\title{
Molecular breeding for transgenic rice with low-phytic-acid phenotype through manipulating myo-inositol 3-phosphate synthase gene
}

\author{
Mio Kuwano $\cdot$ Akio Ohyama $\cdot$ Yusuke Tanaka $\cdot$ \\ Tetsuro Mimura $\cdot$ Fumio Takaiwa . \\ Kaoru T. Yoshida
}

Received: 17 May 2006/ Accepted: 26 July 2006/ Published online: 29 September 2006

(C) Springer Science+Business Media B.V. 2006

\begin{abstract}
In most plant seeds, phosphorus is stored primarily as myo-inositol 1,2,3,4,5,6hexakisphosphate ( $\operatorname{InsP}_{6}$; phytic acid). Reducing the phytic acid content of seeds is a major breeding target, both to increase the availability of mineral nutrients and to decrease the environmental load of phosphorus. The first step in phytic acid biosynthesis and inositol metabolism is catalyzed by 1D-myo-inositol 3-phosphate $\left(\operatorname{Ins}(3) \mathrm{P}_{1}\right)$ synthase. In this study, we aimed to reduce phytic acid levels in rice seeds by manipulating the expression of the rice Ins(3) $\mathrm{P}_{1}$ synthase gene RINO1 using transgenic methods. RINO1 cDNA was transformed into rice plants in the antisense orientation under
\end{abstract}

M. Kuwano $\cdot$ K. T. Yoshida $(\bowtie)$

Graduate School of Agricultural and Life Sciences,

The University of Tokyo, Yayoi 1-1-1, Bunkyo-ku,

Tokyo 113-8657, Japan

e-mail: ayosida@mail.ecc.u-tokyo.ac.jp

A. Ohyama $\cdot$ F. Takaiwa

National Institute of Agrobiological Sciences,

Kannondai 2-1-2, Tsukuba, Ibaraki 305-8602, Japan

Present Address:

A. Ohyama

National Institute of Vegetable and Tea Sciences,

Ano, Mie 514-2392, Japan

Y. Tanaka $\cdot$ T. Mimura

Department of Biology, Faculty of Science, Kobe

University, Rokkodai 1-1, Nada-ku, Kobe, Hyogo

657-8501, Japan the control of the rice major storage protein glutelin GluB-1 promoter. The $\mathrm{T}_{4}$ generation of a stable transgenic line that contained four copies of the transgene showed little morphological differences compared to non-transgenic rice. In the $\mathrm{T}_{5}$ seeds of this line, severe reductions in RINO1 protein levels were observed during the late maturing stages of ripening. Most of the $T_{5}$ seeds contained higher amounts of inorganic phosphates (Pi), without a reduction in total phosphorus levels, compared to non-transgenic seeds. Ion chromatography analysis suggested that the increase in available $\mathrm{Pi}$ is accompanied by a molar-equivalent decrease in phytic acid P. The expression patterns of RINO1 and $G l u B-1$ were examined by quantitative real-time reverse transcriptase-polymerase chainreaction (RT-PCR). Potential strategies for further molecular breeding to reduce phytic acid levels in seeds are discussed.

Keywords Gene expression ·

Glutelin promoter $\cdot$ myo-inositol 3-phosphate synthase - Oryza sativa L. Phytic acid ·

Transgenic plants
Abbreviations
DAF days after flowering
Ins(3) $\mathrm{P}_{1}$ 1D-myo-inositol 3-phosphate
InsP $_{6} \quad$ myo-inositol 1,2,3,4,5,6-
hexakisphosphate
IPK inositol phosphate kinase 
lpa low-phytic-acid

$\mathrm{Pi} \quad$ inorganic phosphate

RT-PCR reverse transcriptase-polymerase chain-reaction

\section{Introduction}

Water pollution caused by human activities, such as agricultural runoff and sewage production, is a serious environmental problem. The influx of excessive phosphorus from fertilizers, human and domestic animal wastes, detergents, and industrial sources can result in the eutrophication of lakes and marshes. Animal waste is a leading source of phosphorus pollution from agricultural sources.

In most cereal grains, phosphorous is stored primarily as the molecule myo-inositol 1,2,3,4,5,6hexakisphosphate ( $\left.\operatorname{InsP}_{6}\right)$, termed phytic acid. Up to $80 \%$ of the total phosphorus in seeds is phytic acid, with the rest consisting of soluble inorganic phosphate (Pi) and the phosphorus present in nucleic acids, proteins, lipids, and sugars. Phytic acid strongly binds to various mineral cations, including potassium, magnesium, calcium, iron, and zinc, to form a mixed salt called phytin (Lott et al. 1995). Monogastric animals utilize phytic acid phosphorus inefficiently because they lack the digestive enzyme phytase, which is required to cleave phosphorus from phytic acid (Cromwell et al. 1993). Mineral phosphate supplements are commonly added to animal diets to prevent phosphorus deficiency, but this practice leads to high phosphorus concentrations in waste matter, contributing to the pollution of water bodies (Sims et al. 2000). Reducing the phytin content of seeds would both increase the availability of mineral nutrients and decrease the environmental phosphorus load.

The first step in phytic acid biosynthesis and inositol metabolism is the formation of $1 \mathrm{D}-$ myoinositol 3-phosphate $\left(\operatorname{Ins}(3) \mathrm{P}_{1}\right)$ from $\mathrm{D}$-glucose 6-phosphate, catalyzed by $\operatorname{Ins}(3) \mathrm{P}_{1}$ synthase. The biochemical pathways from $\operatorname{Ins}(3) \mathrm{P}_{1}$ to $\mathrm{InsP}_{6}$ and the enzymes that catalyze these steps are not fully understood. Yoshida et al. (1999) reported the isolation of a cDNA corresponding to the rice $\operatorname{Ins}(3) \mathrm{P}_{1}$ synthase gene RINO1. They demonstrated that RINO1 transcripts accumulate at high levels in the scutellum and aleurone layer, coinciding well with the appearance of phytin. They suggested that $\operatorname{Ins}(3) \mathrm{P}_{1}$ synthase plays a key role in phytic acid biosynthesis in developing rice seeds.

Reducing the expression of RINO1 in developing seeds may lead to a valuable reduction in the phytin content of seeds. Recently, Feng and Yoshida (2004) attempted to reduce the phytic acid content of rice seeds by manipulating RINO1 expression using transgenic methods. The antisense RINO1 cDNA fused to either the RINO1 promoter or the Cauliflower Mosaic Virus 35S promoter was used to create transgenic rice that produced seeds with a low-phyticacid (lpa) phenotype. Manipulation of RINO1 gene expression led to increases in levels of available $\mathrm{Pi}$ in the transgenic rice seeds but no reduction in the total seed phosphorus levels (Feng and Yoshida 2004). However, the effects of the chimeric transgenes used in the study were not as strong as the effects of the rice lpa mutations reported to date (Larson et al. 2000). The less-pronounced effect of the transgenes was thought to be partly caused by the promoters that were used. GUS expression analysis revealed that the RINO1 promoter, as well as the $35 \mathrm{~S}$ promoter, was active in vegetative tissues other than developing seeds (Feng and Yoshida 2004). Because RINO1 catalyzes the first step in inositol metabolism, as well as phytic acid biosynthesis, the complete repression of RINO1 gene expression in tissues other than seeds is strongly detrimental to plant development. The use of a promoter that is active only in developing seeds, the storage site of phytic acid, may be crucial for obtaining seeds with the lowest possible phytic acid content.

The promoter of the gene for the seed storage protein glutelin, the 1302-bp GluB-1 promoter, is well known to drive extremely high and endosperm-specific expression during rice seed maturation (Wu et al. 1998), and is generally used in transgenic research. Here, we attempted to reduce the phytic acid content of rice seeds by using the $G l u B-1$ promoter to direct RINO1 gene expression in transgenic rice plants. 


\section{Materials and methods}

Plasmid construction and transformation procedures

The antisense RINO1 cDNA, with a length of 1,844 bp (Yoshida et al. 1999), was fused to the rice seed storage glutelin $G l u B-1$ promoter fragment of 1,302 bp (Wu et al. 1998). This construction was inserted into the binary vector pGPTV-35S-HPT, which contains the rice GluB-1 terminator and the hygromycin phosphotransferase gene as a selectable marker (Qu and Takaiwa 2004). The resulting plasmid was transferred into Agrobacterium tumefaciens strain EHA105 by electroporation. Transgenic rice plants (Oryza sativa cv. Kitaake, an early heading variety) were produced by Agrobacterium-mediated transformation. An Agrobacterium harboring the plasmid was used to infect scutellum-derived calli of rice. The regenerated seedlings were selected on media containing hygromycin, transplanted, and grown in a greenhouse as described by Goto et al. (1999).

Selection of transgenic lines

Phosphorus levels in $\mathrm{T}_{1}$ seeds from self-fertilized $\mathrm{T}_{0}$ plants were analyzed as described below. $\mathrm{T}_{1}$ generation lines that contained seeds with $\mathrm{Pi}$ concentrations more than 3-fold greater than seeds of non-transgenic rice were selected, and the seeds that were harvested from the selected lines were planted and grown to produce $\mathrm{T}_{2}$ seeds. These lines were selected because seeds with nearly 2-fold greater amounts than the average concentration of non-transgenic seed $\mathrm{Pi}$ could be observed in the non-transgenic lines, but seeds that showed more than 3-fold greater levels could only be seen in the transgenic seeds. We decided that the 3-fold greater seed Pi concentrations compared to non-transgenic lines would indicate the effect of the transgenes. The number of transgene copies in $T_{1}$ plants was assessed by genomic Southern blot analysis as described below. $\mathrm{T}_{2}$ seeds were only harvested from $\mathrm{T}_{1}$ plants that contained one or more copies of the transgenes. The $\mathrm{T}_{2^{-}}, \mathrm{T}_{3^{-}}$, and $\mathrm{T}_{4}$-generation seeds were similarly analyzed and selected to identify heritable phenotypes. Plant height, number of panicles on $T_{4}$ plants, and weight of 100 dry $T_{5}$ seeds were measured and compared with those of non-transgenic plants.

Genomic Southern blot analysis

Genomic DNA was extracted from rice leaves using the DNeasy Plant Mini Kit (Qiagen, Hilden, Germany). DNA was digested using the restriction endonuclease EcoRI, electrophoresed on a $0.8 \%$ agarose gel, and blotted onto Biodyne A membrane (Pall, Melbourne, Australia). The blotted nucleic acids were fixed by baking at $80^{\circ} \mathrm{C}$ for $2 \mathrm{~h}$.

To produce a template for labeling a DNA probe, a PCR reaction was carried out with the RINO1 cDNA using the upper primer GTGGTGGTGTTGTGGACTGC (position 782 ) and the lower primer AGAATGGGAAAGAC CGAAAA (position 1773). The PCR product was labeled using DIG DNA Labeling Mix (Roche Diagnostics, Basel, Switzerland) to produce a DIG-labeled cDNA probe. The labeling reactions and hybridization procedures were carried out according to the manufacturer's instructions. Detection was performed using the Luminescent Image Analyzer LAS-3000 (Fuji Film, Tokyo, Japan).

Analysis of seed phosphorus levels

Dehusked whole seeds were dried for $48 \mathrm{~h}$ at $60^{\circ} \mathrm{C}$. Each grain was crushed using a Multi-Beads Shocker (Yasui Kikai, Osaka, Japan) and weighed. The Pi was measured from one half of each crushed seed and the total phosphorus from the other half, after which the percentage of Pi in each seed was calculated. To analyze Pi levels, the crushed powder was extracted in $12.5 \%(\mathrm{w} / \mathrm{v})$ trichloroacetic acid containing $25 \mathrm{mM} \mathrm{MgCl}_{2}$, and then centrifuged at $20,000 \times g$ for $10 \mathrm{~min}$. Pi in the supernatant was assayed colorimetrically as described by Chen et al. (1956).

Total phosphorus in the seed was determined as described by Raboy et al. (2000). The total phosphorus was extracted from the crushed powder using the wet-ashing method, followed by a colorimetric assay. 
Ion chromatography analysis of $\mathrm{Pi}$ and phytic acid

Five dehusked whole seeds were gathered and crushed using a Multi-Beads Shocker and weighed. Fifty milligrams of the crushed seeds were ground with a mortar and a pestle and homogenized in $2.4 \%(\mathrm{w} / \mathrm{v}) \mathrm{HCl}$. The homogenates were boiled for $10 \mathrm{~min}$ and centrifuged at $20,000 \times g$ for $10 \mathrm{~min}$ at $4^{\circ} \mathrm{C}$. The supernatant was filtered through a $5.0-\mu \mathrm{m}$ filter (Millipore, Bedford, USA). The filtrate was filtered through a 10000 NMWL filter (Millipore) and was diluted with deionized water to a final concentration of $0.48 \% \mathrm{HCl}$. The $\mathrm{HCl}$-extracts were subjected to ion chromatography as described by Mitsuhashi et al. (2005). This method can separate $\mathrm{InsP}_{1}$ to InsP $_{6}$ in a single gradient elution.

The amount of $\mathrm{Pi}$ or phytic acid in a sample was calculated from a standard curve determined from the peak area of the reference dilution series, from 0 to $3 \mathrm{mg} \mathrm{ml}^{-1}$ for Pi (Phosphorus Std. Soln., 160-19241, Wako, Osaka, Japan) and from $0 \mathrm{mg} \mathrm{ml}^{-1}$ to $30 \mathrm{mg} \mathrm{ml}^{-1}$ for phytic acid (Phytic acid dodecasodium salt from rice, P-3168, Sigma, St. Louis, USA), respectively.

\section{Immunoblot analysis}

Total soluble proteins from dehusked whole seeds collected at 7, 10, 14, 21, and 28 days after flowering (DAF) were weighed and extracted in PBST (PBS, pH 7.4, containing 0.5\% Triton X100). The extract was centrifuged at $20,000 \times g$ for $10 \mathrm{~min}$ at $4^{\circ} \mathrm{C}$, and protein in the supernatant was quantified using the Bio-Rad Protein Assay (Bio-Rad, Chicago, USA) according to the manufacturer's instructions. A $20-\mu \mathrm{g}$ aliquot of protein was loaded onto a $10 \%$ polyacrylamide SDSPAGE gel, separated by electrophoresis, and transferred to Immun-Blot PVDF Membrane (Bio-Rad).

RINO1 protein produced in $E$. coli was used to raise antibodies in a rabbit (Feng and Yoshida 2004). The blotted membranes were incubated with the rabbit anti-RINO1 antiserum, and the RINO1 protein was visualized using a goat antirabbit IgG alkaline phosphatase conjugate using the ProtoBlot II AP System (Promega, Madison,
Wisconsin, USA) according to the manufacturer's instructions. The signals were detected with a LAS-3000 imaging system. Quantitative estimates of the optical densities of the bands were obtained using a Science Lab 2001 Image Gauge (Fuji Film).

\section{Quantitative real-time RT-PCR}

Total RNA from rice (cv. Nipponbare) tissues was extracted from 7-day-old roots and leaves, flowers at one day before anthesis, pistils at flowering, embryos at $10 \mathrm{DAF}$, and dehusked whole seeds at 7, 10,14,21, and 28 DAF using the RNeasy Plant Mini Kit (Qiagen). To eliminate residual genomic DNA, the RNA was treated with DNase I using the RNeasy Micro Kit (Qiagen) according to the manufacturer's instructions.

To quantify the target RNA, in vitro-transcribed RNA was used as a standard. In vitro transcription was performed using the MEGAscript T3 and T7 kit (Ambion, Austin, USA) for RINO1 and GluB1 , respectively. The template DNA was removed using DNase I treatment, and then the transcribed RNA was purified using the RNeasy Mini Kit. After the RNA concentration was determined by spectrometry, the reference RNA was used to create a 10 -fold dilution series, from $100 \mathrm{pg} \mathrm{ml}^{-1}$ to $10 \mathrm{ag} \mathrm{ml}^{-1}$. Real-time RT-PCR amplification was carried out using the QuantiTect SYBR Green RT-PCR Kit (Qiagen) and an ABI PRISM 7700 Sequence Detector (Applied Biosystems, Foster City, USA). The reaction mixture was adjusted to $20 \mathrm{ng}$ total RNA for RINO1 and 2 ng total RNA for $G l u B-1$ in $20-\mu l$ reactions. Real-time RT-PCR was performed using the following primer pairs: RINO1 (5'-CCGTGGCATGTGGCAAAGAG-3' and 5'-TGCATAGCCCGATAAGAGTC-3') and GluB-1 (5'-TATTCTCTCGCCGTTCTGGA-3' and $5^{\prime}$-TCACGCTCTTGCTTTCTTCAA-3'). The annealing temperature was $54^{\circ} \mathrm{C}$, and the PCR reactions were performed in triplicate. Each primer pair generated a single specific RT-PCR product of the expected length. The numbers of RNA molecules were calculated from the linear regression of the standard curve. Data were analyzed using ABI PRISM 7700 Sequence Detection System software (Applied Biosystems). 


\section{Results and discussion}

Production of stable transgenic rice lines

Thirty-six independent hygromycin-resistant transgenic rice plants were generated by Agrobacterium-mediated transformation of a chimeric gene consisting of the rice seed storage protein glutelin $G l u B-1$ promoter and rice RINO1 cDNA in the antisense orientation. All seeds of the transgenic lines showed the same germination rate $(100 \%)$ as the non-transgenic seeds. To examine the storage form of phosphorus in transgenic seeds, we examined available Pi levels in the $\mathrm{T}_{1}$ seeds of individual transgenic lines and compared them to levels in non-transgenic rice seeds. Pi content and total seed phosphorus in non-transgenic rice seeds were almost constant. Higher seed Pi levels were detected in four of the 36 independent $T_{0}$ transgenic lines. Some seeds of these lines had more than three times the level of Pi than non-transgenic seeds. We selected these four transgenic lines (lines 8, 13, 22, and 35) and proceeded to the $T_{4}$ generation.

Chimeric transgenes in transgenic plants

The number of transgene copies in the four $\mathrm{T}_{4}$ transgenic lines was examined by genomic Southern blot analysis. We detected at least five, two, four, and four copies of the transgenes in lines 8, 13, 22, and 35, respectively (Fig. 1). The transgenes in these lines were maintained from the $T_{1}$ to the $T_{4}$ generation, and the Southern hybridization pattern was the same for all $15 \mathrm{~T}_{4}$ plants of each transgenic line irrespective of the presence of multiple transgene copies.

Morphological traits of transgenic plants

We compared the morphology of the $\mathrm{T}_{4}$ plants to that of non-transgenic plants. In juvenile plants, all $\mathrm{T}_{4}$ and non-transgenic rice plants showed similar morphologies. In mature plants, there was little difference in plant height and number of panicles among plants from $\mathrm{T}_{4}$ lines 13 and 22 and non-transgenic plants (Fig. 2a, b; $P=0.05$ ). However, we found significant differences in plant height and number of panicles of the $T_{4}$

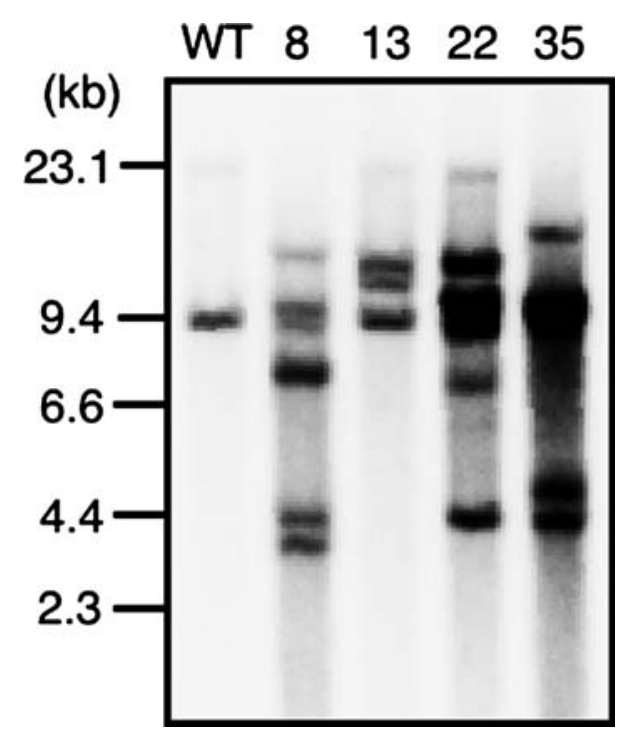

Fig. 1 Southern blot analysis of RINO1. Genomic DNA (3 $\mu \mathrm{g}$ per lane) of non-transgenic lines (WT) and transgenic lines 8, 13, 22, and 35 digested with EcoRI was separated on an agarose gel, blotted, and hybridized with the RINO1 probe. The positions and sizes (in $\mathrm{kb}$ ) of markers are indicated

plants from lines 8 and 35 compared to those of non-transgenic plants. The weight of 100 dry seeds from lines 13, 22, and 35 was not significantly different from that of non-transgenic rice (Fig. 2c; $P=0.05$ ). However, there was a significant difference between seeds from line 8 and non-transgenic seeds. Other morphological traits were similar among non-transgenic and transgenic plants.

Phosphorus levels in transgenic plants

We examined the total phosphorus levels in seeds of non-transgenic rice and $\mathrm{T}_{5}$ seeds of transgenic lines (Fig. 3a). The average total phosphorus content in $\mathrm{T}_{5}$ seeds from lines $8,13,22$, and 35 and non-transgenic seeds was 3.2, 3.2, 2.9, 2.7, and $2.8 \mathrm{mg} \mathrm{g}^{-1}$, respectively. No significant difference was observed among lines 22 and 35 and the nontransgenic seeds $(P=0.05)$. To examine the storage form of phosphorus in the seeds, available $\mathrm{Pi}$ levels in non-transgenic seeds and $\mathrm{T}_{5}$ seeds of transgenic lines were determined (Fig. 3b). The $\mathrm{Pi}$ content in individual grains was determined. $\mathrm{Pi}$ concentrations in non-transgenic seeds were 

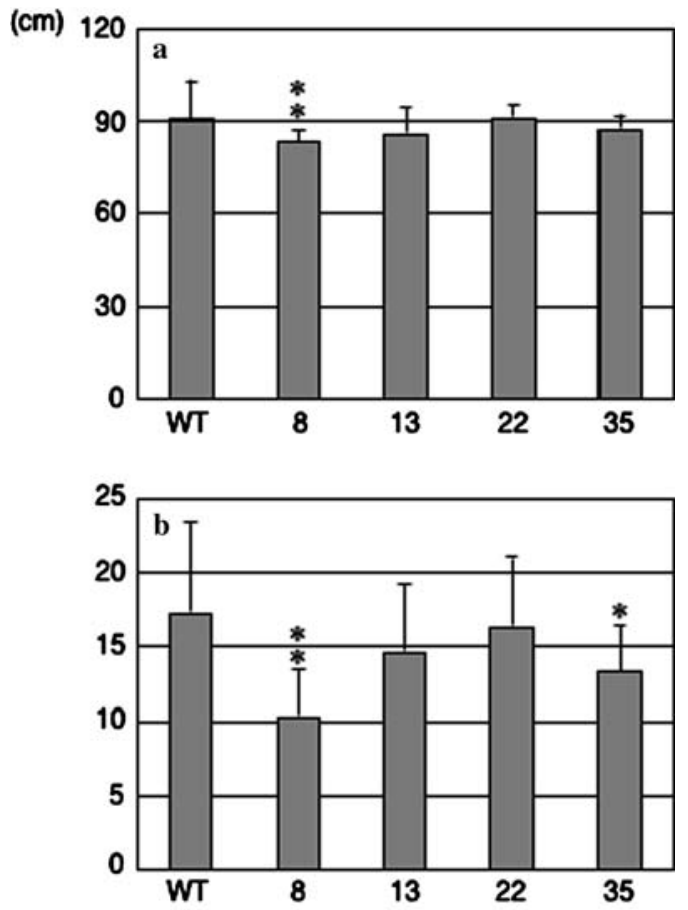

(g)

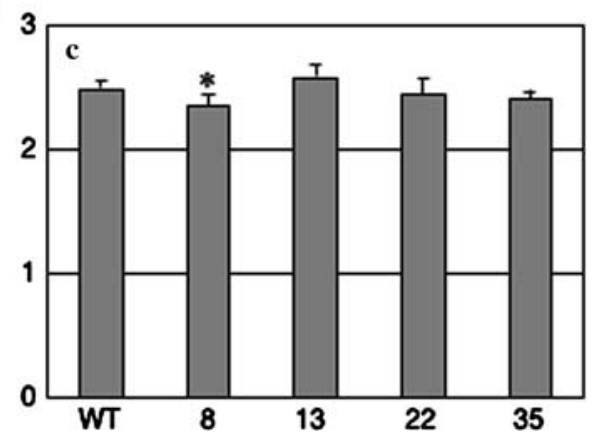

Fig. 2 Plant height (a), number of panicles (b), and weight of 100 dry seeds (c) of non-transgenic rice (WT) and the transgenic rice lines $8,13,22$, and 35 . The values shown are the means $\pm \mathrm{SE}$ of 15 (for $\mathbf{a}$ and $\mathbf{b}$ ) or 5 (for $\mathbf{c}$ ) independent measurements. The symbols * and $* *$ indicate significant differences at $P=0.05$ and 0.01 , respectively, when compared to non-transgenic plants

$0.065-0.11 \mathrm{mg} \mathrm{g}^{-1}$, and constituted $2.5-3.0 \%$ of the total phosphorus in each seed. Seeds of lines 13, 22, and 35 had greater Pi concentrations than non-transgenic seeds, with $\mathrm{Pi}$ constituting an average of $8.8,16$, and $9.0 \%$ of the total phosphorus, respectively. The highest Pi concentration in this experiment, $1.4 \mathrm{mg} \mathrm{g}^{-1}$, was observed in a seed of line 22. In this seed, the Pi content corresponded to $48 \%$ of the total phosphorus (Fig. 3b).
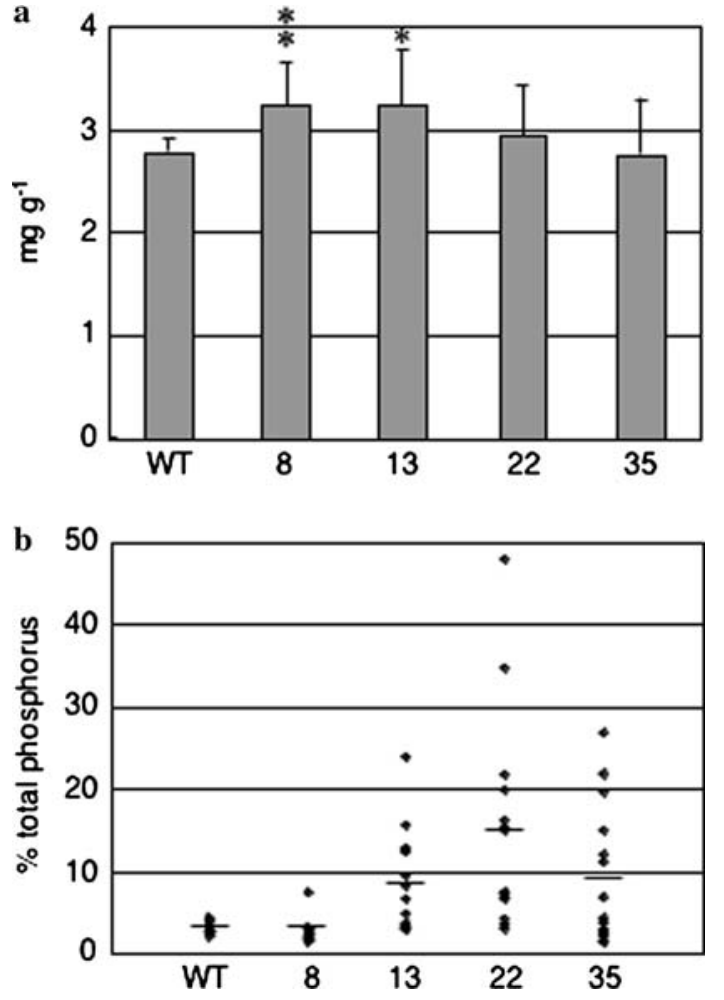

Fig. 3 Total phosphate concentrations (a) and Pi fraction of the total phosphorus (b) in non-transgenic seeds (WT) and transgenic $\mathrm{T}_{5}$ seeds of lines $8,13,22$, and 35. Each total phosphorus value represents the mean \pm SE of 15 seeds. The symbols $*$ and $* *$ indicate significant differences at $P=0.05$ and 0.01 , respectively, when compared to nontransgenic plants. The Pi content of each seed is shown as a percentage of the total phosphate content. Horizontal lines indicate mean values

To quantify phytic acid and Pi levels, seed extracts from the transgenic line 22 and the non-transgenic line were analyzed by ion chromatography. Line 22 was chosen for examination in further studies because there were no significant differences in morphological traits (Fig. 2), and the highest Pi level was found in this line (Fig. 3). Larger conductivity of $\mathrm{Pi}$ and smaller conductivity of phytic acid was detected for the seeds of line 22 compared to the seeds of the nontransgenic plants (Fig. 4a). Mean phytic acid values was $18 \mu \mathrm{mol} \mathrm{g}^{-1}$ for the non-transgenic seeds, and $15 \mu \mathrm{mol} \mathrm{g}^{-1}$ for the transgenic line (Fig. 4b). This represented an average reduction in seed phytic acid content of $17 \%$ for line 22 . Ion chromatography analysis also confirmed that no accumulations of other inositol phosphates are 


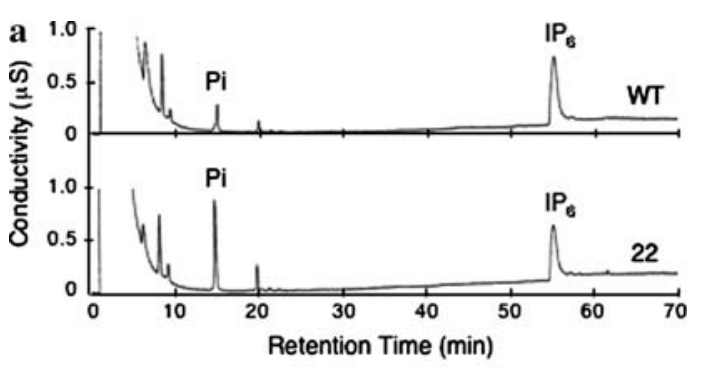

b
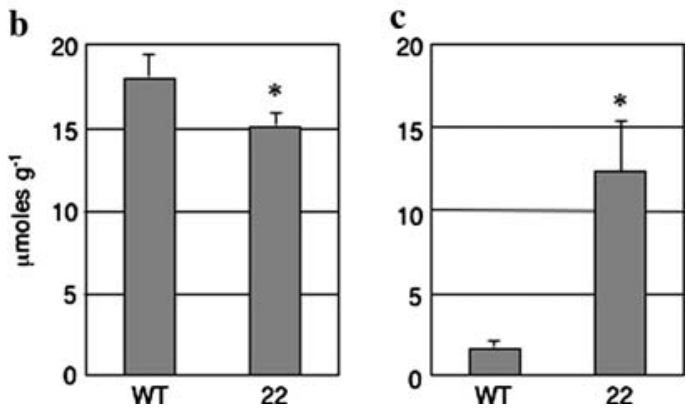

Fig. 4 Ion chromatography analysis of phytic acid and Pi content in non-transgenic seeds and transgenic $\mathrm{T}_{5}$ seeds of line 22. (a) Typical chromatograms of extracts from nontransgenic seeds and transgenic $\mathrm{T}_{5}$ seeds of line 22. WT, non-transgenic seeds; 22 , transgenic $\mathrm{T}_{5}$ seeds of line 22 . Fifty milligrams of tissue from five seeds were extracted, and equal aliquots of filtered supernatant were fractionated. (b, c) Concentrations of phytic acid (b) and $\mathrm{Pi}(\mathbf{c})$ in non-transgenic seeds and transgenic $\mathrm{T}_{5}$ seeds of line 22 . Each value represents the mean \pm SE for six replicates. The symbol $*$ indicate significant differences at $P=0.05$ when compared to non-transgenic plants

observed in the transgenic seeds. Pi content in transgenic seeds were $12 \mu \mathrm{mol} \mathrm{g}^{-1}$ compared to $1.5 \mu \mathrm{mol} \mathrm{g}^{-1}$ from non-transgenic plants indicating that expression of transgenes during seed development resulted in an 9-fold increase in $\mathrm{Pi}$ (Fig. 4c). In non-transgenic seeds, phosphorus in the phytic acid form was $108 \mu \mathrm{mol} \mathrm{g}^{-1}$ because phytic acid is equivalent to $\mathrm{InsP}_{6}$ and has six phosphates. Phytic acid form $\mathrm{P}$ decreased to $90 \mu \mathrm{mol} \mathrm{g}^{-1}$ in transgenic seeds, while the available $\mathrm{P}$ increased to $12 \mu \mathrm{mol} \mathrm{g}^{-1}$ compared to $1.5 \mu \mathrm{mol} \mathrm{g}^{-1}$ in non-transgenic plants. There was no significant difference in the amount of seed total $\mathrm{P}$ between line 22 and non-transgenic line (Fig. 3a). These results indicate that the reduction in phytic acid $\mathrm{P}$ is accompanied by a molarequivalent increase in $\mathrm{Pi}$.

The results of this study indicate the potential feasibility of using molecular breeding to alter the storage form of phosphorus in seeds through the manipulation of RINO1 gene expression under the control of a strong seedspecific promoter. In the rice lpa mutant, $\mathrm{Pi}$ represents $32 \%$ of the total seed phosphorus (Larson et al. 2000). Although a large variation in $\mathrm{Pi}$ concentration was observed in transgenic seeds (Fig. 3b) and on average the effects of the transgenes used in this study were not so great as the effects of the lpa mutations, the grain with the highest Pi level (48\%) in line 22 suggests that transgenic lines with much greater rice grain Pi contents could be obtained through molecular breeding.

In some cereal plants, the position in a panicle affects the growth rate of a seed. The timing and the level of expression of some genes encoding starch biosynthesis were affected by the position in a panicle of rice (Ishimaru et al. 2005). In this study, differences in expression patterns of the transgenes at different positions in a panicle may be a major reason for the large variation in $\mathrm{Pi}$ concentrations observed in the transgenic plants.

The RINO1 protein in transgenic seeds

Expression of the RINO1 gene in transgenic plants was assessed using immunoblotting. Total proteins of immature $\mathrm{T}_{5}$ seeds from line 22 and non-transgenic plants at $7,10,14,21$, and 28 DAF were subjected to immunoblotting analysis using anti-RINO1 antiserum. The RINO1 polyclonal antibody reacted with a band of about $56 \mathrm{kDa}$. In non-transgenic seeds, the RINO1 protein was detected from $7 \mathrm{DAF}$, and its level increased to a maximum at $10 \mathrm{DAF}$, after which the level decreased (Fig. 5a). No significant difference was seen in RINO1 protein levels of maturing seeds from 7 to 14 DAF between $\mathrm{T}_{5}$ seeds from line 22 and non-transgenic seeds (Fig. 5b, c). In contrast, a decreased level of RINO1 protein compared to that in nontransgenic seeds was observed after 14 DAF. The decreased level of RINO1 protein suggests that the expression of the antisense RINO1 transcripts efficiently represses RINO1 gene expression later in the ripening period in line 22 . The immunoblot analysis demonstrated that 

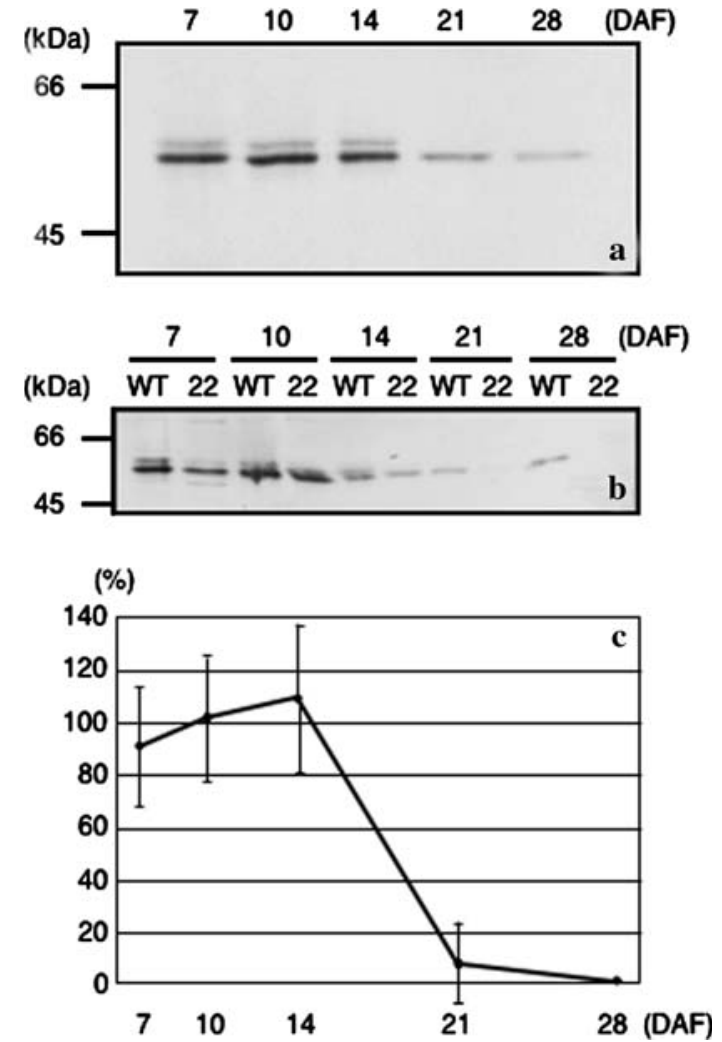

Fig. 5 Immunoblot analysis of RINO1 protein in nontransgenic seeds and transgenic $\mathrm{T}_{5}$ seeds of line 22. (a) Immunoblot analysis of non-transgenic seeds. Each lane contains $20 \mu \mathrm{g}$ of protein from individual seeds at 7, 10, 14, 21, and $28 \mathrm{DAF}$. The positions and sizes (in $\mathrm{kDa}$ ) of markers are indicated. (b) A typical result of nontransgenic seeds (WT) and transgenic $\mathrm{T}_{5}$ seeds of line 22. Each lane contains $20 \mu \mathrm{g}$ of protein from an individual seed at 7, 10, 14, 21, and 28 DAF. (c) Relative amounts of RINO1 protein in $T_{5}$ seeds of line 22 . Each time point represents the relative image density of RINO1 protein bands on immunoblots as a percentage of that in nontransgenic seeds. Each value represents the mean $\pm \mathrm{SE}$ for nine replicates

increased levels of $\mathrm{Pi}$ in the transgenic line may be related to decreased levels of the RINO1 protein during the late stages of ripening.

\section{Expression patterns of RINO1 and GluB-1}

To determine the promoter activities and to evaluate a strategy for further increase in the seed available $\mathrm{Pi}$, the relative levels of RINO1 and GluB-1 transcripts in non-transgenic rice were estimated by quantitative real-time RT-PCR. High levels of RINO1 transcripts were observed

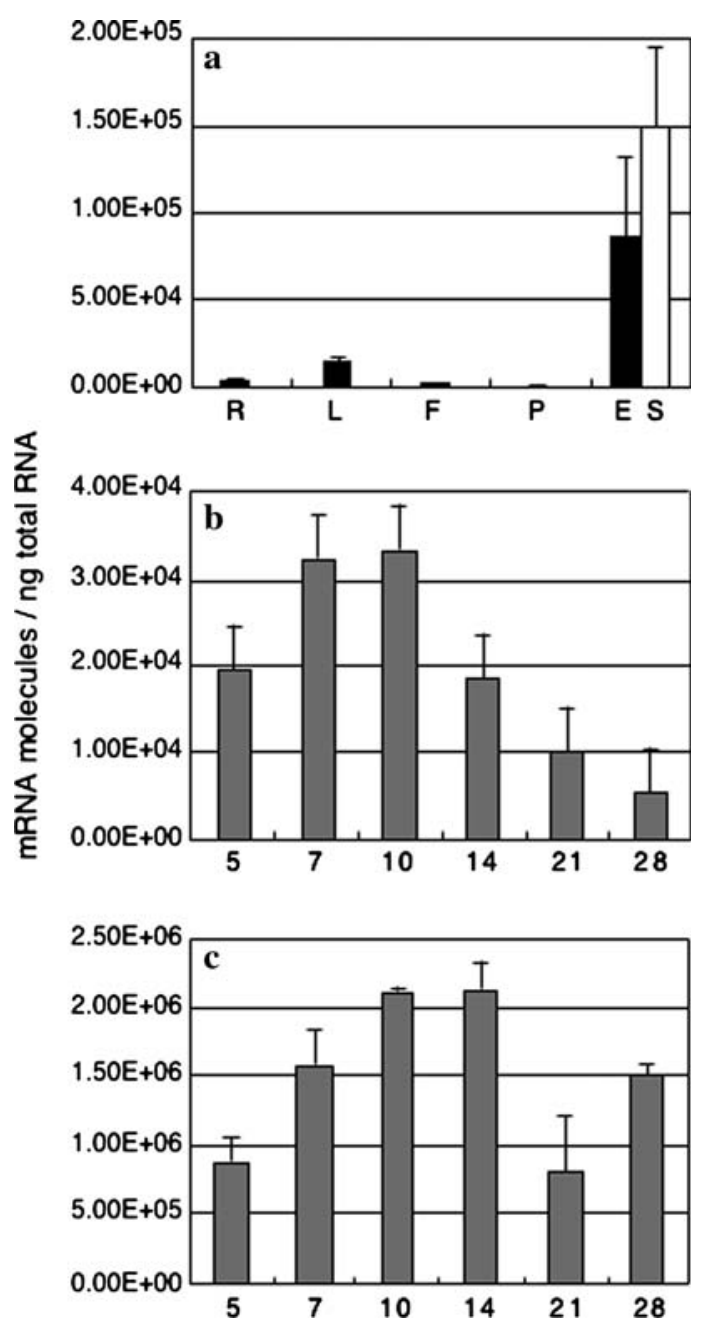

Fig. 6 Quantitative real-time RT-PCR analysis of RINO1 and $G l u B-1$ in non-transgenic seeds. (a) mRNA levels of RINO1 (solid bars) and GluB-1 (open bars) in roots (R) and leaves (L) at 7 days after germination, flowers $(F)$ at 1 day before flowering, pistils $(\mathrm{P})$ at flowering, and embryos (E) and dehusked whole seeds (S) at 10 DAF. $(\mathbf{b}, \mathbf{c )}$ mRNA levels of RINO1 (b) and GluB-1 (c) in dehusked whole seeds at $5,7,10,14,21$, and $28 \mathrm{DAF}$

in developing embryos, in contrast to the low levels observed in roots, leaves, flowers, and pistils (Fig. 6a). Glu B-1 transcripts were exclusively observed in seeds and the expression level was extremely high. This result agrees well with the seed-specific property of the promoter of the seed storage protein gene $G l u B-1$, and indicates the potential of this promoter to drive extremely high expression in endosperm (Wu et al. 1998). During seed maturation, RINO1 mRNA was detected 
from 5 DAF and increased to a maximum at 7-10 DAF, after which the level decreased (Fig. 6b). This result corresponds well with immunoblot results for the RINO1 protein in non-transgenic seeds (Fig. 5a). The maximum GluB-1 transcript occurred from 10 to 14 DAF (Fig. 6c). This difference in the temporal patterns of RINO1 and GluB-1 transcript accumulation (mRNA levels) may partially account for the difficulty in the complete suppression of the inherent RINOI transcript by the antisense RINO1 cDNA driven by the GluB-1 promoter (Fig. 5c). RINO1 is expressed at high levels within the scutellum and the aleurone layer, where phytic acid is stored (Yoshida et al. 1999), whereas expression of theGluB-1 promoter, as revealed by GUS activity, was initially detected in the aleurone and the subaleurone layers, and then in the endosperm (Qu and Takaiwa 2004). This spatial difference in expression may be another reason for the limited repression of the RINO1 gene in transgenic rice.

In this study, we demonstrated the effect of the seed specific promoter $G l u B-1$ on producing high-Pi seeds. In transgenic line 22, the Pi level was on average about 5-fold higher than in nontransgenic plants. The highest Pi value of line 22 was about 16 -fold greater than that of nontransgenic plants, and was about 1.5 -fold greater than in the rice lpa mutant. These results indicate the possibility of molecular breeding for rice with an ultra lpa phenotype through the manipulation of RINO1 gene expression. Stronger effects could be obtained by using a promoter with the same temporal and spatial activity in the seed as RINO1, thereby reducing the levels of RINO1 protein earlier in the process of seed maturation.

An alternative approach that has recently been reported to generate grains with lpa phenotype is the seed-specific disruption of inositol phosphate kinases (IPKs). Loss-of-function mutants of $\mathrm{Ara}$ bidopsis thaliana that disrupted either AtIPK1 or AtIPK $2 \beta$ exhibit reductions in seed phytic acid levels (Stevenson-Paulik et al. 2005). We have isolated the rice orthologs of IPK1 and IPK2, and are now working on characterizing them. We are planning to investigate whether rice IPK genes can be used in the genetic engineering of lpa phenotype seeds.
Another strategy that has been reported earlier to achieve the same goal as our research is the expression of Aspergillus phytase in transgenic rice grains (Lucca et al. 2001). This approach was aimed to degrade seed phytic acid when the grains were fed to animals, but not in planta. To obtain the most effective results, these transgenic cereals are suggested to be ground to powder, and incubated at $37^{\circ} \mathrm{C}$ with water (Drakakaki et al. 2005). These preparation steps for crop feed would not be necessary when seeds with lpa phenotype are used. Molecular breeding of rice grains with decreased phytic acid content would be a promising strategy to achieve high effects with low cost and labor.

Acknowledgments The authors are grateful to Drs. N. Tsutsumi and M. Nakazono for helpful discussions. This work was supported by a Grant-in-Aid for Scientific Research from the Ministry of Education, Science, Sports and Culture of Japan to K.T.Y. (15380004) and by a grant from the Ministry of Agriculture, Forestry, and Fisheries of Japan (Rice Genome Project) to K.T.Y.

\section{References}

Chen PS, Toribara TY, Warner H (1956) Microdetermination of phosphorus. Anal Chem 28:1756-1758

Cromwell GL, Stahly TS, Coffey RD, Monegue HJ, Randolph JH (1993) Efficacy of phytase in improving the bioavailability of phosphorus in soybean meal and corn-soybean meal diets for pigs. J Anim Sci 71:18311840

Drakakaki G, Marcel S, Glahn RP, Lund EK, Pariagh S, Fischer R, Christou P, Stoger E (2005) Endospermspecific co-expression of recombinant soybean ferritin and Aspergillus phytase in maize results in significant increases in the levels of bioavailable iron. Plant Mol Biol 59:869-880

Feng X, Yoshida KT (2004) Molecular approaches for producing low-phytic-acid grains in rice. Plant Biotechnol 21:183-189

Goto S, Yoshihara T, Shigemoto N, Toki S, Takaiwa F (1999) Iron fortification of rice seed by the soybean ferritin gene. Nature Biotechnol 17:282-286

Ishimaru T, Hirose T, Matsuda T, Goto A, Takahashi K, Sasaki H, Terao T, Ishii R, Ohsugi R, Yamagishi T (2005) Expression patterns of genes encoding carbohydrate-metabolizing enzymes and their relationship to grain filling in rice (Oryza sativa L.): comparison of caryopses located at different positions in a panicle. Plant Cell Physiol 46:620-628

Larson SR, Rutger JN, Young KA, Raboy V (2000) Isolation and genetic mapping of a non-lethal rice 
(Oryza sativa L.) low phytic acid 1 mutation. Crop Sci 40:1397-1405

Lott JNA, Greenwood JS, Batten GD (1995) Mechanisms and regulation of mineral nutrient storage during seed development. In: Kigel J, Galili G (eds), Seed development and germination. Marcel Dekker, New York, pp 215-235

Lucca P, Hurrell R, Potrykus I (2001) Genetic engineering approaches to improve the bioavailability and the level of iron in rice grains. Theor Appl Genet 102:392397

Mitsuhashi N, Ohnishi M, Sekiguchi Y, Kwon YU, Chang YT, Chung SK, Inoue Y, Reid RJ, Yagisawa H, Mimura T (2005) Phytic acid synthesis and vacuolar accumulation in suspension-cultured cells of Catharanthus roseus induced by high concentration of inorganic phosphate and cations. Plant Physiol 138:1607-1614

Qu LQ, Takaiwa F (2004) Evaluation of tissue specificity and expression strength of rice seed component gene promoters in transgenic rice. Plant Biotechnol J 2:113-125
Raboy V, Gerbasi PF, Young KA, Stoneberg SD, Pickett SG, Bauman AT, Murthy PPN, Sheridan WF, Ertl DS (2000) Origin and seed phenotype of maize low phytic acid 1-1 and low phytic acid 2-1. Plant Physiol 124:355-368

Sims JT, Edwards AC, Schoumans OF, Simard RR (2000) Integrating soil phosphorus testing into environmentally based agricultural management practices. J Environ Qual 29:60-71

Stevenson-Paulik J, Bastidas RJ, Chiou ST, Frye RA, York JD (2005) Generation of phytate-free seeds in Arabidopsis through disruption of inositol polyphosphate kinases. Proc Natl Acad Sci 102:12612-12617

Wu CY, Adachi T, Hatano T, Washida H, Suzuki A, Takaiwa F (1998) Promoters of rice seed storage protein genes direct endosperm-specific gene expression in transgenic rice. Plant Cell Physiol 39:885-889

Yoshida KT, Wada T, Koyama H, Mizobuchi-Fukuoka R, Naito S, (1999) Temporal and spatial patterns of accumulation of transcript of myo-inositol-1-phosphate synthase and phytin-containing particles during seed development in rice. Plant Physiol 119:65-72 